Methods We describe the clinical presentation, results of laboratory and radiological investigations, treatment and outcome to date.

A review of current available literature on this topic was also undertaken.

Results An 8 year old boy presented to the PED with severe nausea and vomiting with a cyclical pressure type right upper quadrant pain for 7 hours. On presentation the pain had reduced significantly. No symptoms of infection, no concerning vomit contents, diarrhoea or constipation.

This was the 10th similar episode in the previous 2 months. Previous investigations including blood panel, urine were normal and symptoms had resolved on attendance.

No abnormality was found on clinical exam.

Abdominal ultrasound demonstrated a large right sided hydronephrosis secondary to PUJO confirmed by CT KUB. A renogram demonstrated a partial obstruction and surgical management was planned electively.

Conclusion Our patient had experienced multiple episodes of Dietl's Crisis which had resolved independently. PUJO is not a common first time presentation in children of this age. We suggest that Paediatricians consider this diagnosis when the other more common differentials have been outruled while being mindful that clinical examinations, radiological and labaroratory investigations may be normal in between episodes of Dietl's crises.

\section{P571 FOETAL FEMUR FRACTURE AND EXTERNAL CEPHALIC VERSION: A CASE REPORT OF METAPHYSEAL INJURY AND LITERATURE REVIEW}

Dragos Valceanu*, Gerry Mackin, Patrick Mcllwaine, Isabela Govor. South West Acute Hospital, Enniskillen, UK

\subsection{6/archdischild-2019-epa.905}

Introduction External Cephalic Version (ECV) is a procedure used to turn a foetus from a breech or transverse position into a cephalic position prior to the onset of labour. It is recommended by the UK national guidelines to enable vaginal delivery for breech presentation of a singleton pregnancy. ECV is considered a safe manoeuvre when dealing with breech presentation and fractured femur is a rare but recognised complication of this procedure, with only 3 cases reported in the speciality literature.

Case We herein report a 4 months old boy, born at $38+4 / 40$ weeks gestational age by Kiwi extraction and episiotomy, with a birth weight of $4180 \mathrm{~g}$, Apgar score 8 at 1 minute and 9 at 5 minutes of life, no active resuscitation was required. The pregnancy was carefully monitored due to the maternal nutritional controlled gestational diabetes and foetopelvic unstable lie. Was breech at $37+5$ weeks and ECV manoeuvre performed, discharged home in stable condition afterwards. Postnatally paediatric team or parents did not raise any concern regarding pain or swelling of the knee. He was admitted to our Children's Ward at 10 days of age by a community midwife with suspected non-accidental injury. Physical examination revealed right lower limb in a flexed, antalgic position, non-ecchymotic swelling of the right knee, with tenderness to touch. Full examination revealed no additional injuries. Retrospective review of pictures taken by parents in the first day of life revealed a swollen knee being held in a flexed position.
Imaging investigations X-Ray Right knee reported florid callus formation surrounding the distal femoral metaphysis, representing an ossifying subperiosteal haematoma along with a bony fragment in relation to the anterolateral aspect of the distal metaphysis in keeping with an avulsion fracture.

Conclusion Based on antenatal, perinatal and postnatal history, revision of maternal case notes, and photographic evidence while being inpatient in the maternity ward, along with the presence of callus formation on X-Ray implied an injury older than 10 days. This suggests that the femoral fracture is most likely due to External Cephalic Version performed 6 days prior to delivery. A decision was made by the paediatric consultant to withhold any further safeguarding investigations as an aetiology for the child's fracture was detected.

\section{P572 PARTIAL DOUBLE TRISOMY 9 AND 13-FIRST REPORTED CASE IN MEDICAL LITERATURE}

${ }^{1}$ Qasim Mahmood, ${ }^{2,3}$ Birendra Rai ${ }^{*},{ }^{4}$ David Betts, ${ }^{1}$ Rizwan Khan. ${ }^{1}$ University Hospital Kerry, Kerry, Ireland; ${ }^{2}$ Mullingar Regional Hospital, Mullingar, Ireland; ${ }^{3}$ Northampton General Hospital, Northampton, UK; ${ }^{4}$ Our Lady's Children's Hospital, Crumlin, Ireland

\subsection{6/archdischild-2019-epa.906}

Background Both trisomy $9 p$ and partial trisomy $13 q$ have been recognised in past with characteristics clinical anomaly, our case is the first reported case of combined partial double trisomy involving chromosome $9 \mathrm{p}$ and $13 \mathrm{q}$. Phenotypic Characteristics vary based on the regions of the chromosome involved and the gene dosages effect. Characteristics of our index case would not only help clinician in genotype-phenotype correlation of any such future cases but would also add up to the already described consequences in offspring of balanced reciprocal translocations in either parents

Case report A female infant was born at 41 weeks gestation by normal delivery with birth weight $3.3 \mathrm{kgs}$. The pregnancy was uneventful. Baby had an episode of hypoglycaemia during very first day of life.

Physical examination of the baby revealed profound central hypotonia, head lag, low set ears, depressed nasal bridge and increased nuchal pad of fat. Cardiac examination revealed soft systolic murmur of grade $2 / 6$ which subsequently on echocardiography was noted to arise from a small atrial septal defect. Remainder systemic examination was within normal limits. Further course in the special care baby unit was complicated by recurrent apneas, desaturations and poor feeding. She also developed symptoms of cow milk protein's intolerance and gastro oesophageal reflux later on in life. Cranial ultrasound, Electroencephalography, MRI of the brain, renal ultrasound, sleep study, Laryngo bronchoscopy, chest and thoracic inlet Xrays were all normal. Array comparative genomic hybridisation, using a $60 \mathrm{~K}$ Agilent chip showed a gain of chromosome 9 material of approximately $30.9 \mathrm{Mb}$ at bands $9 \mathrm{p} 24.3-9 \mathrm{p} 21.1$ between base pair coordinates 204193 and 31104204, and an another gain of chromosome 13 material of approximately $11.2 \mathrm{Mb}$ at bands $13 \mathrm{q} 12.11-13 \mathrm{q} 12.3$ between base pair coordinates 20407295 and 31578124 , with the former representing the most proximal probe on this platform. Subsequent analysis of $G$ banded metaphase chromosomes demonstrated an abnormal female karyotype with an additional chromosome consistent with a der(13)t $(9 ; 13)(\mathrm{p} 21.1 ; \mathrm{q} 12)$.

Karyotypic analysis of the parents showed that the mother carried a balanced $t(9 ; 13)$ translocations. Therefore the transferred genetic defect in the index case was a product of $3: 1$ 
segregation error of maternal reciprocal translocation $t(9 ; 13)$ (p21;q12).

Conclusion Balanced reciprocal translocations in either parents can amplify and produce unbalanced gamets leading to defective conceptus. Prenatal diagnosis is strongly recommended where balanced translocation is found in parent. Clinical features of the affected conceptus depends largely on the regions of chromosome involved.

\section{P573 A CASE OF INSIDIOUS RECURRENT ABDOMINAL PAIN}

${ }^{1}$ Anita Spirito, ${ }^{1,2,3}$ Enrica Manca*, ${ }^{4}$ Claudio Carmine Guida, ${ }^{1}$ Angela Maggio, ${ }^{5}$ Maria Savino, ${ }^{4}$ Filippo Aucella, ${ }^{3}$ Massimo Pettoello-Mantovani, ${ }^{1}$ Saverio Ladogana. 'Department of Pediatrics, Onco-Hematology Unit, 'Casa Sollievo della Sofferenza' Scientific Institute, San Giovanni Rotondo, Foggia, Italy; ${ }^{2}$ Residency Program in Pediatrics, University of Foggia, Foggia, Italy; ${ }^{3}$ Department of Pediatrics, Pediatric Unit, 'Casa Sollievo della Sofferenza' Scientific Institute, San Giovanni Rotondo, Foggia, Italy; ${ }^{4}$ Department of Nephrology and Dialysis- Interregional Reference Center for the Prevention, Surveillance, Diagnosis and Treatment of Porphyria, 'Casa Sollievo della Sofferenza' Scientific Institute, San Giovanni Rotondo, Foggia, Italy; ${ }^{5}$ mmmunogenetic Laboratory- Department of Transfusion Medicine and Analysis Laboratory, 'Casa Sollievo della Sofferenza' Scientific Institute, San Giovanni Rotondo, Foggia, Italy

10.1136/archdischild-2019-epa.907

Porphyrias are a group of inherited metabolic disorders of heme biosynthesis leading to excessive accumulation and excretion of porphyrins.The variable clinical manifestations may determine a delay in the diagnosis, followed by a possible negative clinical outcome.

We describe a case of a 14-year-old girl with ShwachmanDiamond syndrome (SDS) and epilepsy admitted with severe cyclic abdominal pain not responsive to antalgic therapy, localized in the epigastric area, irradiating to the whole abdomen and the back, associated to aspecific symptoms (diarrhea, fever,vomiting). During hospitalization, the girl was asymptomatic. We excluded infectious, autoimmune, endocrinological causes, a relapse of SDS and performed a screening for porphyria that resulted negative. After each attack, she referred presenting urinary retention followed by hyperchromic urines, anxiety and paresthesias. Past clinical data showed hyponatremia, tachycardia and hypertension. Eventually, increased values of ALA $(8.21 \mathrm{mg} / \mathrm{l})$ and PBG $(3.96 \mathrm{mg} / \mathrm{l})$ were found; exposure of fresh urines to sunlight caused a change of their color. Finally, genetic analysis was negative. These findings allowed the diagnosis of porphyria, therefore we prescribed a normocalorichyperglucidic diet andpreventive glucose solutions in stressing situations, with regression of symptoms.

Acute porphyrias present with life-threatening crisis secondary to the injury of central, peripheral and autonomic nervous system. They can be triggered by drugs, alcohol, infections, reduced caloric intake, endogenous hormone cycles and stressing situations. The characteristic manifestations are severe cyclic abdominal pain, neurological or psychiatric symptoms and/or hyponatremia. Diagnostic is the assessment of plasmatic PBG/ALA, always increased during an attack, normal during remission. Treatment is human haemin although, in mild attacks, a diet with high carbohydrates and/or preventive glucose infusions is effective; a negative genetic evaluation should never exclude the diagnosis.

It is important considering acute porphyria in the differential diagnosis of severe cyclic abdominal pain, particularly in subjects affected by different and/or rare clinical disorders.

\section{P574 COMPLEX REGIONAL PAIN SYNDROME AND HPV VACCINE: A CASE REPORT OF COMPLEX REGIONAL PAIN SYNDROME AND LITERATURE REVIEW}

Isabela Govor*, Gerry Mackin, Dragos Valceanu. South West Acute Hospital, Enniskillen, UK

\subsection{6/archdischild-2019-epa.908}

Introduction Complex Regional Pain Syndrome (CRPS) is a chronic pain disorder characterised by significant autonomic features which typically develops in an extremity after acute tissue trauma. Symptoms may include continuing pain, sensory abnormalities like allodynia,hyperalgesia,hypoesthesia,vasomotor abnormalities in skin colour or temperature, sudomotor abnormalities of sweating or oedema and motor/trophic abnormalities in hair,skin,nails, tremor or muscle weakness.HPV vaccination is offered to all girls il Northern Ireland aged 14/ 15 years and recent plans have been made to offer it to boys starting late 2019. Since the introduction of HPV vaccination there have been case reports across the world describing various side effects after HPV vaccination, including Complex Regional Payn Syndrome. One report alone detailed 45 patients across 13 countries. In Japan the vaccination was temporarily halted as a consequence. The VaccineAdverseEventReportingSystem(VAERS) USA, has received 31.911 reports of adverse events following any HPV vaccination from which 22 $(0.07 \%)$ reports met the criteria for complex regional pain syndrome (including reflex sympathetic dystrophy) as an adverse event. 21 cases were after Gardasil (HPV4) vaccine and 1 was after Cervarix (HPV2) vaccine.

Case We report a previously fit healthy 14 years old Irish girl. She had received her 2nd HPV (Gardasil) vaccine on 09/04/ 18 having received her 1 st vaccine without any problems in October 2017. Twenty minutes post vaccine her left arm from the elbow down became swollen, purple and painful.Presentation was consistent, CRPS which was initially not diagnosed at first presentation to ED and Paediatrics. Correct diagnosis made four weeks post event and appropriate treatment initiated with full recovery withing eight months.

Laboratory investigations No significant abnormalities.

Conclusion There are documented cases of CRPS after HPV vaccine although is still controversial as to whether it is a vaccine or the effect of the trauma associated with inserting a needle which is the aetiological factor. This case highlights the need for better recognition of this condition amongst paediatricians as earlier initiation of treatment is associated with better long term outcome.

\section{P575 CASE REPORT: TWINS WITH NIEMAN PICK SYNDROME}

Samy AA Allawendy*, Cormac Duff, Dara Gallagher, Ann Sheehan. Sligo University Hospital, Sligo, Ireland

\subsection{6/archdischild-2019-epa.909}

Introduction Niemann-Pick Disease is an autosomal recessive metabolic disorder characterized by deposition of sphingomyelin and cholesterol within the lysosome of reticuloendothelial cells of various organs due to inherited deficiency of an enzyme, acid sphingomyelinase. Niemann-Pick Disease is classified into four types such as A, B, C and D each caused by a different gene mutation.

Case We report a case of Niemann Pick Disease type A/B twins. The patients are 3 years old girls, who presented to Sligo University Hospital with repeated episodes of chest 